\title{
Expression levels of iron homeostasis genes predict liver graft tolerance
}

Most solid organ transplant recipients require life-long administration of immunosuppressants. Occasionally, some recipients develop spontaneous graft tolerance, that is, they are able to maintain stable graft function without receiving immunosuppressive therapy. However, the mechanisms by which this tolerance occurs are not fully understood.

Felix Bohne and colleagues investigated the molecular pathways associated with graft tolerance and rejection in liver transplant recipients in a prospective, multicenter immunosuppression withdrawal clinical trial. The study included 75 patients, 33 of whom tolerated their grafts and discontinued immunosuppressive therapy; the other 42 rejected their grafts (nontolerant).

The researchers analyzed gene expression levels in blood and liver tissue samples. Interestingly, the main difference between tolerant and nontolerant patients was in the set of genes involved in regulating iron homeostasis. In particular, the gene that encodes hepcidin (the master regulator of iron metabolism) was overexpressed in samples taken from patients who tolerated their grafts. Serum levels of hepcidin and ferritin were also increased in these patients.

Moreover, in an independent set of patients, gene expression measurements were able to accurately predict the outcome of immunosuppressive drug withdrawal. Bohne et al. speculate that iron metabolism has a critical role in graft tolerance and rejection.

Isobel Franks

Original article Bohne, F. et al. Intra-graft expression of genes involved in iron homeostasis predicts the development of operational tolerance in human liver transplantation. J. Clin. Invest. 122, 368-382 (2012) 